\title{
Do Electrons and Ions Coexist in an EBIT?
}

\author{
A. Lapierre ${ }^{1}$, J. R. Crespo López-Urrutia ${ }^{1}$, J. Braun ${ }^{1}$, G. Brenner ${ }^{1}$. H. \\ Bruhns $^{1}$, D. Fischer ${ }^{1}$, A. J. González Martínez ${ }^{1}$, V. Mironov ${ }^{1}$, C. J. \\ Osborne $^{1}$, G. Sikler ${ }^{1}$, R. Soria Orts ${ }^{1}$, H. Tawara ${ }^{1}$, Y. Yamazaki ${ }^{2}$, J. \\ Ullrich $^{1}$ \\ ${ }^{1}$ Max-Planck-Institut für Kernphysik, Saupfercheckweg 1, D-69117 Heidelberg, Germany \\ ${ }^{2}$ RIKEN, Hirosawa 2-1, Wako-shi, Saitama 351-01, Japan
}

\begin{abstract}
Detailed analysis of an extended exponential tail observed in the decay curve of the ${ }^{2} \mathrm{P}_{3 / 2}{ }^{2} \mathrm{P}_{1 / 2}$ magnetic dipole (M1) transition in boron-like $\mathrm{Ar}^{13+}$ provides evidence that electrons and ions might coexist in the same spatial region in the Heidelberg Electron Beam Ion Trap (HD-EBIT). On this basis, new trapping-cooling-recombination schemes for positron-antiproton plasmas are envisioned, integrated in a magnetic bottle configuration that should be able to trap the subsequently formed recombined cold antihydrogen. Moreover, the EBIT configuration, providing excellent spectroscopic access to the trapping region via seven view ports is shown to be well suited for performing precision spectroscopy of antiprotonic ions. Those might be generated either by recombination of antiprotons with neutral gas atoms or through radiative recombination and state-selective dielectronicrecombination-like processes with highly charged ions produced and stored in the EBIT simultaneously in a nested trap configuration.
\end{abstract}

\section{INTRODUCTION}

Coexistence of antiprotons $(\bar{p})$ and positrons $\left(\mathrm{e}^{+}\right)$in the same volume of space is one essential prerequisite for antihydrogen $(\bar{H})$ formation in a trap. In present machines this is approximated by first confining $\bar{p}$ and $\mathrm{e}^{+}$in separate but nested Penning traps in a homogeneous magnetic field environment and then forcing the positrons to repeatedly pass through the electron-cooled $\bar{p}$ cloud at some velocity (hot or cold mixing) [1, 2]. Recombination and $\bar{H}$ formation has been successfully observed either by imaging $\bar{H}$ annihilation products at the walls of the trap [2] or by detecting $\bar{p}$ produced via field-ionization of $\bar{H}$ transported through a region of a strong electric field [1]. In all present scenarios, $\bar{H}$ is not trapped and its existence is only demonstrated by destroying it again. Thus, substantial efforts are undertaken to combine such recombination schemes with magnetic gradient field geometries that could confine cold $\bar{H}$ after its formation which will be the next decisive step towards high-precision spectroscopy, the ultimate goal of such experiments. Several recombination and trapping scenarios have been and are still being discussed in order to reach that goal, among them, sophisticated "cusp-shaped" traps in an antiHelmholtz configuration [3], laser-induced schemes in $\bar{p}-\mathrm{e}^{+}$plasmas and laserinduced recombination involving positronium, which was successfully demonstrated recently [4].

In this paper we provide evidence that electrons and $\mathrm{Ar}^{13+}$ ions have been confined simultaneously in the same volume of space inside the Heidelberg Electron Beam Ion 
Trap (HD-EBIT). As schematically depicted in the upper part of Figure 1, low-energy electrons might be trapped either by the positive ion space charge potential (ISCP) created by the confined ions or by the axial magnetic bottle potential (dotted line in the upper part of Figure 1) close to the trapping volume of the ions, which are confined axially by electrostatic potentials applied to the drift tubes of the trap (full line in the upper part of Figure 1) as well as radially by the strong magnetic field.
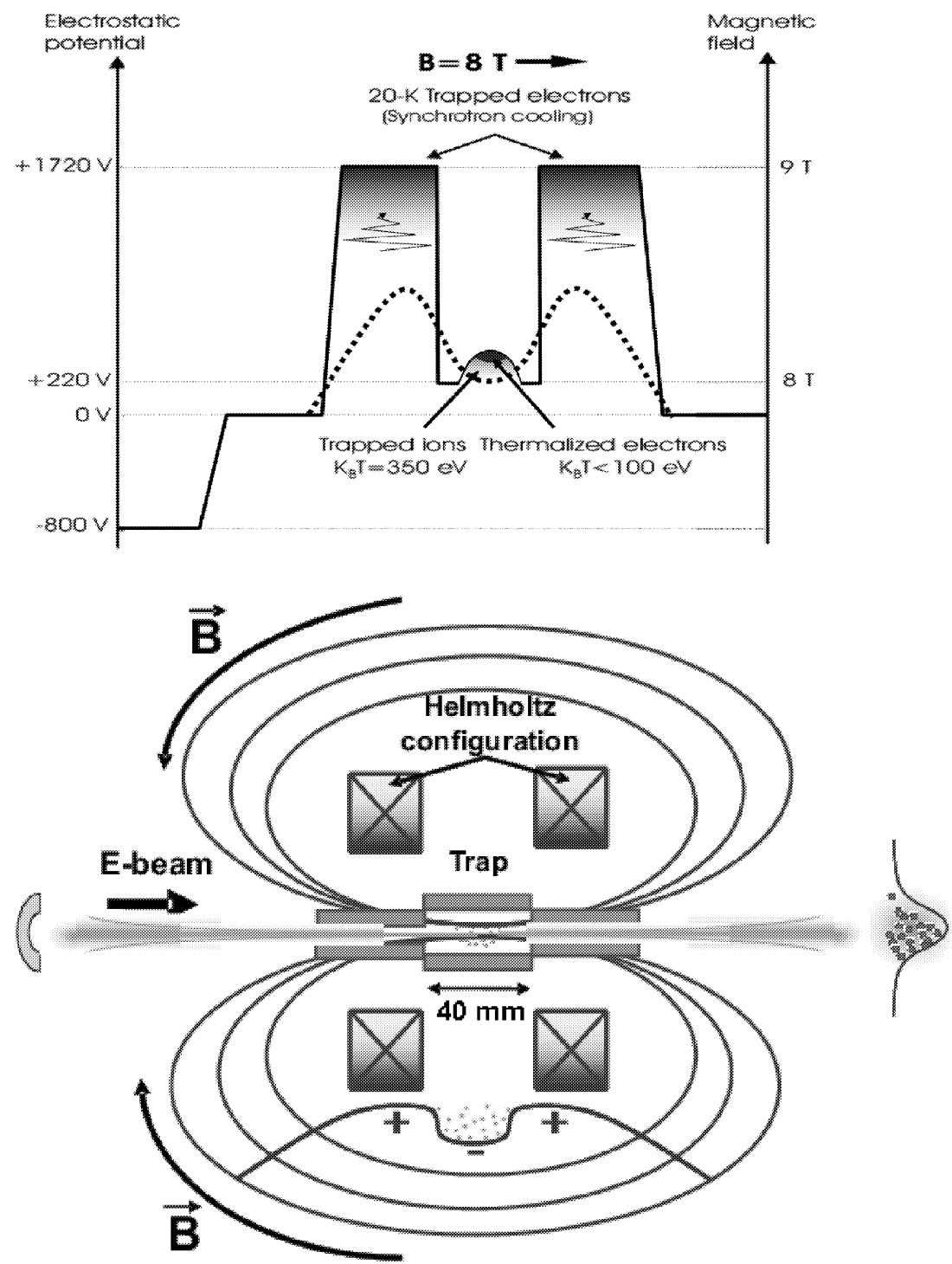

FIGURE 1. Schematic layout of the HD-EBIT (lower part) with superconducting coils, the electron beam, drift tubes with applied electrostatic potentials (full line, upper part) and magnetic bottle configuration (dotted line, upper part). 


\section{LIFETIME MEASUREMENTS AT THE HD-EBIT}

The HD-EBIT along with the on-going physics programme has been described in detail elsewhere [5]. In short, a high-energy electron beam of up to $E_{e}=100 \mathrm{keV}$ at currents as large as $I_{e}=500 \mathrm{~mA}$ is collinearly injected along the Helmholtz-type magnetic field reaching $9 \mathrm{~T}$ at the centre of the trap. The B-field strongly compresses the electron beam in the radial direction to a diameter of less than $100 \mu \mathrm{m}$ thus achieving current densities close to $10^{4} \mathrm{~A} / \mathrm{cm}^{2}$. Ions, efficiently produced by sequential electron-impact ionisation of atoms injected into the trap via a collimated gas jet are radially trapped by the electron beam space charge potential (ESCP), which can reach several hundreds of volts. Longitudinally they are confined by applying appropriate potentials to the various drift tubes (nine in the present set-up). Two experimental view ports, six in next generation machines, provide optimum access for installation of various spectrometers covering essentially all wavelengths of interest. Under present conditions, ions in any charge-state up to helium-like $\mathrm{Hg}^{78+}$ can be created, trapped, cooled via evaporative cooling and investigated inside the trap as well as extracted out of the trap in order to perform collision experiments with atoms, molecules, clusters, and surfaces. In the experiment to be discussed in this contribution, the electron beam current was typically set to $100 \mathrm{~mA}$, the electron beam energy was about $700 \mathrm{eV}$ (below threshold for ionization of $\mathrm{Ar}^{13+}$ ) and the magnetic field was $8 \mathrm{~T}$ in the centre of the trap.

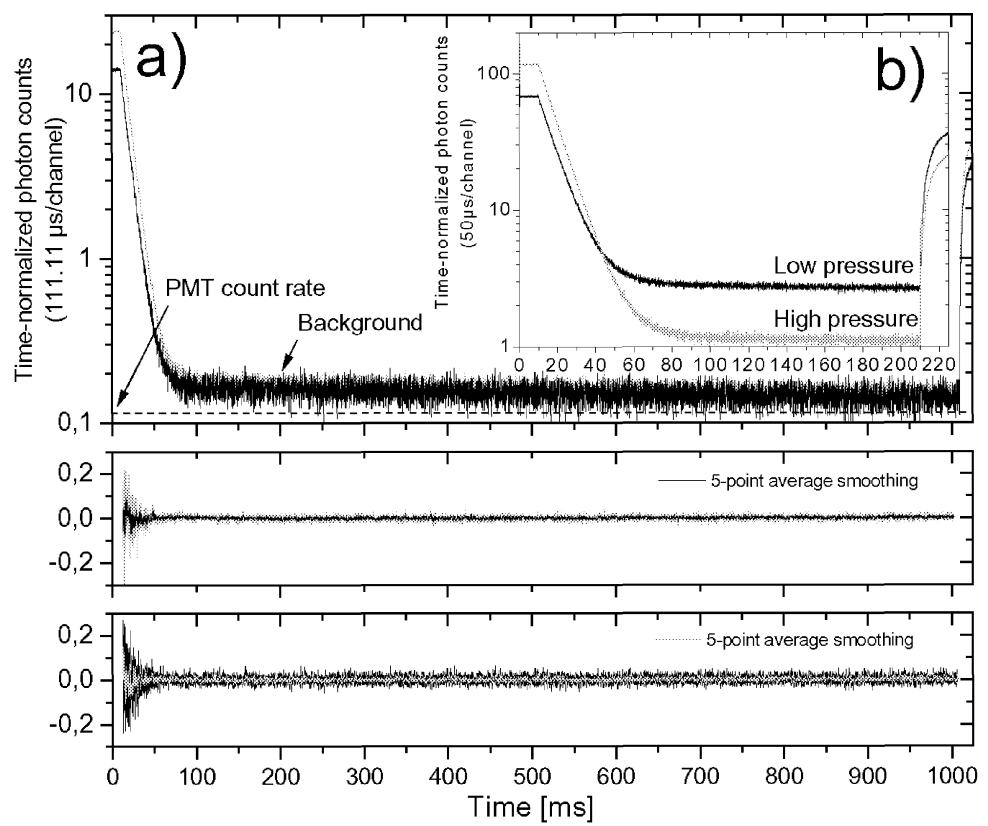

FIGURE 2. Decay curves of the ${ }^{2} \mathrm{P}_{3 / 2}{ }^{2} \mathrm{P}_{1 / 2}$ metastable $\mathrm{M} 1$ transition in boron-like $\mathrm{Ar}^{13+}$ taken for an electron beam switch-off time of a) $1000 \mathrm{~ms}$ and b) $200 \mathrm{~ms}$ for two different injection gas pressures (see text). Lower part: residuals after fitting to a linear combination of two exponential functions. 
As described in detail in Ref. [6], the goal of the present experiment was to measure the lifetime of the ${ }^{2} \mathrm{P}_{3 / 2}$ metastable level of $\mathrm{Ar}^{13+}$. For hat purpose, the electron beam was periodically switched off for time intervals of up to one second and the photons of the M1 transitions, filtered by an interference window, were detected by a photomultiplier tube (PMT) ( $c a .34$ counts/s dark rate). In a previous experiment the energy of the M1 transition was measured to be $441.2559(1) \mathrm{nm}$ with a $5 \cdot 10^{-7}$ accuracy providing the most sensitive test of five-electron quantum electro dynamic (QED) contributions whose overall effect is estimated to be $0.96 \mathrm{~nm}$ [7].

Typical lifetime curves taken at two different Ar gas injection pressures are shown in Figure 2. In addition to the expected fast decay of the metastable level a slowly decreasing background with an apparent lifetime between one to two seconds was observed by fitting the observed decay curve to a linear combination of two exponentials. As demonstrated and discussed in detail in Ref. [6] and as becomes obvious from the residuals from such a fit shown in the lower part of Figure 2, this background did not influence our lifetime measurement beyond the experimental error bar stated in Ref. [6] which is dominated by other sources of uncertainty.

\section{BACKGROUND LEVEL AND TRAPPED ELECTRONS}

Essentially four mechanisms might be responsible for the observed background: (i) ion-ion collisional excitation, (ii) charge-exchange collisions or (iii) cascade repopulation, both feeding the metastable level from upper excited states, and (iv) electron- impact excitation of the metastable level by low-energetic Penning electrons. Excitation by ion-ion collisions (i) or feeding of the metastable level through ion-atom charge-exchange encounters (ii), can both be ruled out since the slowly decaying background level was found to be lower for a higher injection gas pressure as shown in Figure 2. Moreover, the ion temperature estimated from the measured Doppler broadening of the spectral line to be about $350 \mathrm{eV}$ was too low for any highly charged ion to come close enough for efficient ion-impact excitation of the level, i.e., for reaction (i) to occur. Feeding of the metastable level through charge-exchange collisions of $\mathrm{Ar}^{14+}$ with rest gas atoms, reaction (ii), can additionally be ruled out since the $\mathrm{Ar}^{14+}$ abundance in the trap was negligibly small by keeping the electron beam energy safely below the ionization threshold of $\mathrm{Ar}^{13+}$. Cascade repopulation, process (iii), can be discarded since the decay times of all known higher lying levels that could feed the metastable state are less than a few $\mu$ s.

Thus, we conclude that reaction (iv), namely electron-impact excitation of the 2.8 eV M1 transition by low-energy electrons $\left(E_{e}<100 \mathrm{eV}\right)$, trapped in the same volume of space as the ions for even long time periods after the electron beam was switched off, are the reason for the slowly decaying background contribution. In order to substantiate this result we have investigated the behaviour of the background level as a function of several parameters. In Figure 3, for example, we explore the background photon count rate for various "left-over" electron beam current in "switched-off" phases. In other words, the electron beam was not completely turned off, but reduced from the "switched-on" current of $100 \mathrm{~mA}$ to values of $40 \mathrm{~mA}$ or less, as indicated in Figure 3. For "switched-off" electron beam currents above $20 \mathrm{~mA}$ we observed the 
expected behaviour, namely a background level that is larger than the one for $0 \mathrm{~mA}$ and which increases with increasing "switched off" current due to the ongoing currentdepended continuous electron impact excitation of the metastable level by the left-over electron beam. Most surprisingly, however, further reduction of the electron beam to currents of $10 \mathrm{~mA}$ or less, decreased the background level substantially by nearly one order of magnitude for $1 \mathrm{~mA}$ below the background level observed when the electron beam was completely turned off.

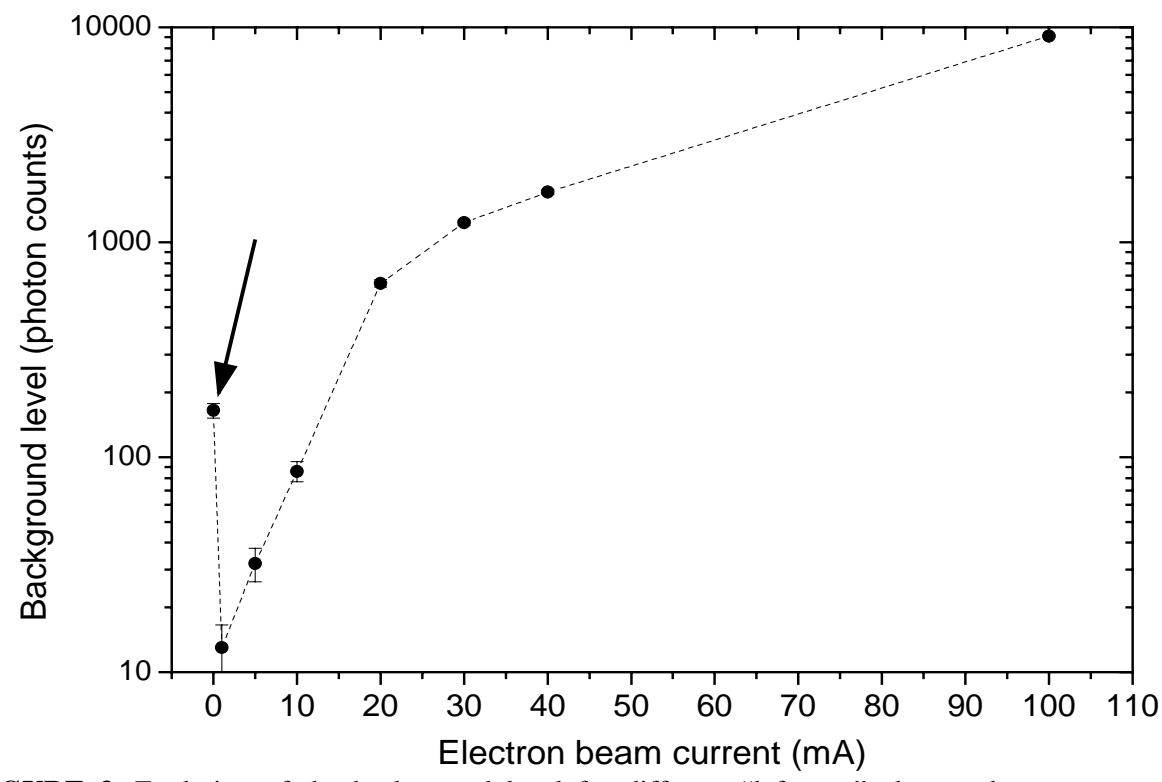

FIGURE 3. Evolution of the background level for different "left-over" electron beam currents in "switched-off" position (see text). The background level observed at $10 \mathrm{~mA}$ and less was lower than that observed at $0 \mathrm{~mA}$, i.e., without electron beam current (see arrow).

At first glance, one might interpret this observation to be a result of a depletion of the $\mathrm{Ar}^{13+}$ population by electron impact ionization at lower currents due to an effective simultaneous enhancement of the electron beam energy above the $\mathrm{Ar}^{13+}$ ionization threshold. Decreasing the current of the electron beam decreases its space charge potential (ESPC), and as a result, increases the beam energy, which can then be sufficient to ionize $\mathrm{Ar}^{13+}$ ions. For instance, at a beam current of $1 \mathrm{~mA}$, the beam energy is increased to about $1 \mathrm{KeV}$. However, simulation calculations of electron capture, electron-impact excitation and -ionization have shown that for beam currents lower than about $20 \mathrm{~mA}$, depletion by electron impact ionization is insignificant because of its squared dependence on the electron beam current.

Therefore, we are led to interpret the observed suppression of the background to be a consequence of a reduction of the number of simultaneously trapped low-energy electrons being pushed away by the space charge of the left-over weak electron beam. This conclusion is further supported by inspecting the decay time of the slowly decreasing background part, extracted from the two-exponential fit. It is found to be 
considerably longer for "switched-off" currents between a few to $20 \mathrm{~mA}$ compared to zero. In most cases indeed, it is compatible with an infinite lifetime within the error bars of the fit, providing additional evidence that excitation by slow trapped electrons is getting less efficient since less such electrons are around if the electron beam is not completely switched off. Above a "switched-off" current of $20 \mathrm{~mA}$, the whole decay curve cannot be fitted any more by a sum of two exponential functions which is consistent with the expectation, that now, in addition to the possible excitation by slow electrons, direct excitation by the left-over electron beam plays an increasing role with increasing "switched-off" current. Indeed, a three-exponential fit delivers increasing decay times of the background rate with higher left-over current, again becoming consistent with infinity for large currents due to a continuous ongoing excitation once a new equilibrium is reached.

Furthermore, we have systematically investigated the behaviour of the background level as a function of the injection gas pressure as indicated in Figure 2 and observed a decreasing background photon count rate with increasing pressure. For higher injection gas pressure a larger numbers of ions is created and confined in the trap as is clearly seen from the increased photon rate at high pressure when the electron beam is switched on. An increased number of stored ions enhances the ISCP and, thus, the potential well depth and the temperature of trapped low-energy electrons (see section four). Since the excitation cross section decreases with increasing electron velocity the background level might indeed expected to be lower in agreement with the observations.

In summary, we conclude that low-energy Penning electrons simultaneously trapped in the same volume in space as the ions are intimately related to the observed slowly decaying background.

\section{LOW-ENERGY ELECTRON TRAPPING}

As illustrated in Figure 1, low-energy electrons which are in general always present in the apparatus could be trapped near the location of the ion cloud after the electron beam was switched off by essentially two mechanisms:

First, they might be confined axially by the magnetic bottle potential at the borders of our $40 \mathrm{~mm}$ trap. Due to the specific geometrical arrangement of the Helmholtz coils in our EBIT, an $8 \mathrm{~T}$ magnetic field at the centre of the trap is increased by about $5 \%$ to $8.4 \mathrm{~T}$ at an axial distance of $26 \mathrm{~mm}$ away from the trap centre with a maximum gradient at $\pm 14 \mathrm{~mm}$ off centre. Accidentally this nearly coincides with the edge of the closest drift tubes and, thus with the axial border of the ion trap. Hence, it can be estimated, neglecting plasma and collision effects (see Ref. [8]), that electrons that are born in the middle of the trap with a pitch angle larger than about $\alpha>77^{\circ}$ are reflected before they could fall into the deep positive potential wells on both sides of the centre drift tubes, which axially confine the ion cloud.

Second, they might be trapped by the ISCP, a scenario seemingly supported for three reasons: (i), even a weak residual electron beam with a current of $1 \mathrm{~mA}$ providing a negative ESCP of only about $-5 \mathrm{~V}$ at the edge of the electron beam radius, about half of which is compensated by the ISCP to an effective value of not more than 
$-3 \mathrm{~V}$, is able to efficiently push away the low-energy electrons from the trapping region. (ii), as shown in Figure 4, increasing the trapping potential for the ions (see inset for the geometry) and, thus the number of trapped ions increased the background level indicating that the ISCP is directly correlated with the number of electrons overlapping with the ion cloud. (iii), whereas the lifetime of the metastable level was found to be independent of the drift tube potential for values higher than about $+300 \mathrm{~V}$, the background decay time decreased continuously with increasing the ion trapping potentials up to values as large as $2.7 \mathrm{kV}$. Thus, the background decay time seems to essentially reflect electron losses from the trap as a function of the ion trapping potential, which are expected to become larger for tighter confinement for the ions, i.e., higher trapping potentials for the electrons outside the ion cloud.

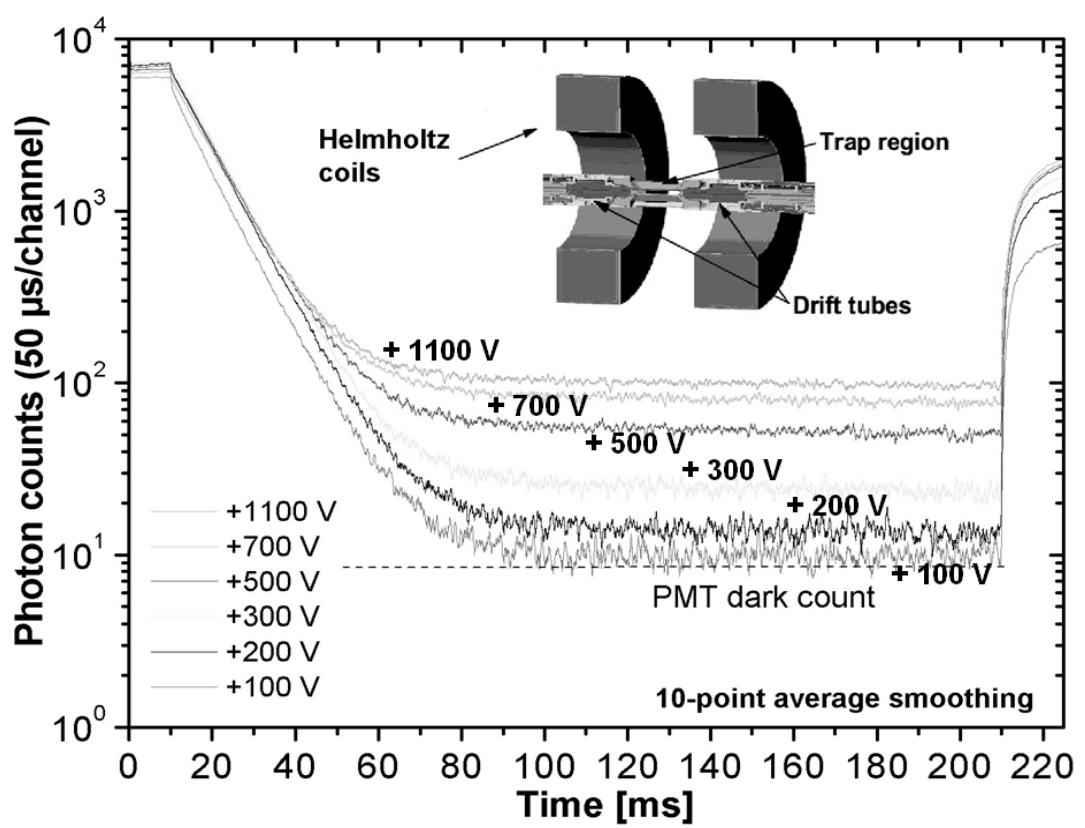

FIGURE 4. Increase of the slowly decaying background level as a function of the drift tube trapping potential as indicated in the Figure.

Whereas we cannot come to a final conclusion about the question on the dominant reason for trapping low-energy electrons on the basis of the present data, confinement of electrons by the positive space charge of the trapped ion cloud (ISCP) seems to be the more likely scenario and was further investigated by the following procedure. The sum space charge potential of both the electron beam and the trapped ion cloud was obtained by measuring the energy position of the maximum fluorescent signal for a given electron beam current and a given potential difference between the cathode and the whole drift tube assembly (the position of this maximum corresponds to an electron beam energy having the ionization potential of $\mathrm{Ar}^{13+}$ ). Estimating theoretically the ESCP, the ISCP alone is found to be about $+250 \mathrm{~V}$, which is enough to trap electrons of sufficiently low energy. As described in detail in Ref. [6], the 
temperature of the low-energy trapped electron $k_{B} T_{e}$ is estimated to be $k_{B} T_{e}=25-125 \mathrm{eV}$ using $\omega=q V / k_{B} T_{e}$ ( $q$ : charge of the trapped particles, $V$ trapping potential, $k_{B}$ : Boltzmann constant), where the dimensionless ratio $\omega$ is ranging from 2 to 10 .

For an average electron temperature of $75 \mathrm{eV}$ and from the measured background count rate we estimate the density of trapped electrons to be $5 \cdot 10^{7} \mathrm{~cm}^{-3}$ assuming a Maxwellian-averaged rate coefficient for electron-impact excitation of the metastable level. The ion density is calculated approximately to be $3 \cdot 10^{9} \mathrm{~cm}^{-3}$ from the measured total number of trapped ions extracted in an independent experiment, and taking the known length of the trap of $40 \mathrm{~mm}$ as well as an ion cloud of about $0.7 \mathrm{~mm}$ in diameter measured previously by an imaging optical spectrometer and by imaging the cloud on a CCD camera. The total numbers of electrons and ions are then estimated to be $\mathrm{N}_{\mathrm{e}}=8 \cdot 10^{5}$ and $\mathrm{N}_{\mathrm{i}}=5 \cdot 10^{7}$, respectively. Also, the equilibrium temperature of the ions under continuous electron bombardment at $\mathrm{I}_{\mathrm{e}}=100 \mathrm{~mA}$ for a $2.5 \mathrm{kV}$ drift tube trapping potential is deduced from the measured Doppler width of the spectral line to be about $k_{B} T_{i}=350 \mathrm{eV}$.

As indicated in Figure 1, low-energy electrons outside the ion cloud rapidly cool down by emission of synchrotron radiation with a cooling time of $56 \mathrm{msec}$ in an $8 \mathrm{~T}$ magnetic field. Thus, one might expect that after a short while, electrons will no longer have sufficient energy to excite the trapped ions, and consequently, the shape of

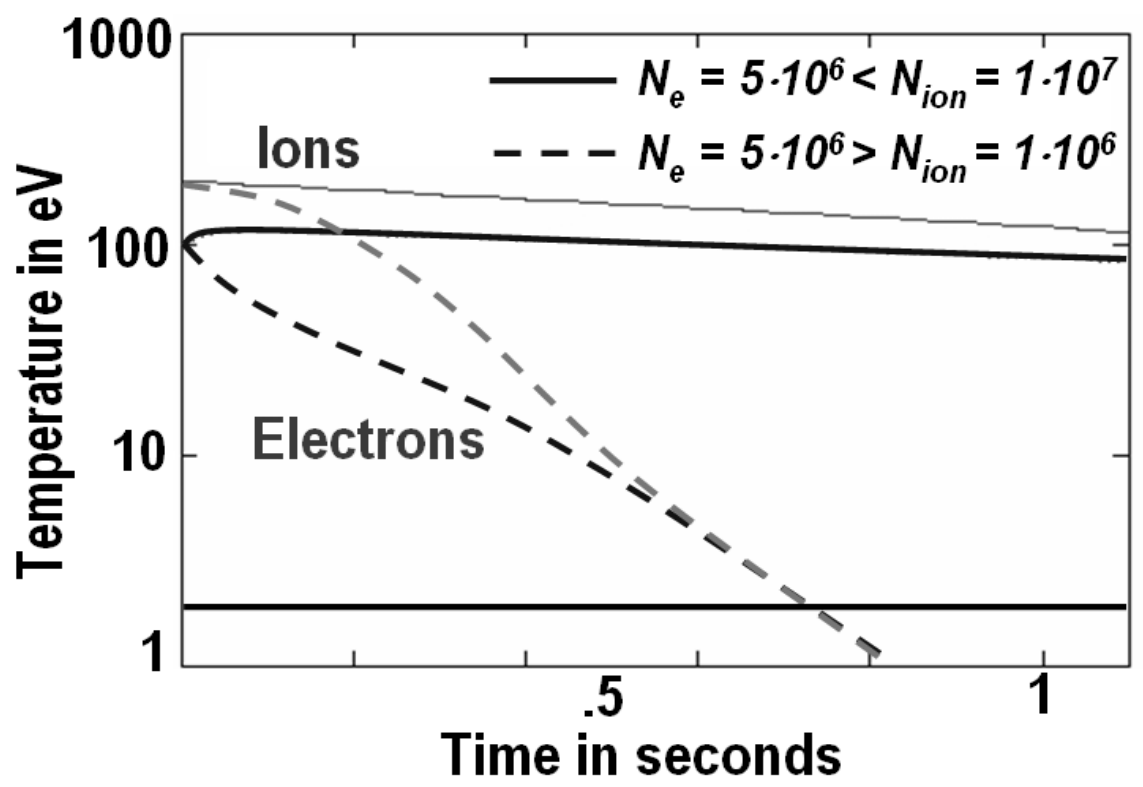

FIGURE 5. Temperature of electrons (dark lines) and ions (grey lines) as a function of time for two different scenarios (dashed and full lines, respectively) of relative numbers of trapped particles. In the present experiment, the ion-to-electron number density ratio is about 60 . 
the exponential background decay curve should abruptly change. Inspection of the decay behaviour by fitting a two-exponential function to 100 times the lifetime did not show any effect as can be seen from the residuals in Figure 2. Calculations presented in Figure 5, using the Spitzer equation [9] with different ratios between the numbers of trapped electrons and ions, show that for a number of ions (dark full line) exceeding that of electrons (grey full line) by only a factor of two (a factor of 60 in the present experiment) the trapped ions act as effective heat sink for the electrons. Thus, electrons and ions are nearly in thermal equilibrium, and they cool down only very slowly such that the metastable level can be excited for seconds and more, as observed.

\section{PROSPECTS AND CONCLUSION}

In the future, several tests will be performed in order to substantiate our present findings and to clarify what might be the main mechanism for trapping low-energy electrons in the volume of space where the ions are confined. In a first experiment one could look for high-energy radiation from radiative recombination of the trapped electrons with the ions after turning off the electron beam even though the recombination rate is so small that estimates for the above numbers of electrons and ions in the trap yield an observable x-ray rate of only $10^{-4}$ per second at an assumed electron temperature of $50 \mathrm{eV}$. Since the recombination rate depends on the square root of the temperature, it should be enhanced for the decreased temperatures of the plasma after longer trapping times and might then become observable. Second, we are presently preparing a YAG pumped dye laser for laser spectroscopy of trapped ions which could be used for stimulating radiative recombination into high-lying levels of the ions as it has been observed in cooler sections of storage rings [10] and proposed as a promising way to enhance radiative recombination in an $\bar{H}-\mathrm{e}^{+}$plasma. Third, with one of our newly designed traps it is straightforward to extract the trapped electrons and measure their number as a simple proof of their existence. Fourth, it will be very instructive to image the ion cloud via the M1 transition radiation by opening the entrance slits of our optical grating spectrometer to get an insight into the steadystate situation of the trapping region with the electron beam on. By switching off the electron beam, this will provide detailed information on the development in space of the ion cloud excited by the low-energy trapped electrons and, thus, give us direct information on their location and expansion in the trap along with the evolution in time of the electron and ion clouds when they cool down.

To conclude, we have provided the first evidence that low-temperature electrons might coexist with highly charged ions in an EBIT configuration. The available extended data sets indicate that the positive space charge potential of the trapped ion cloud might be mainly responsible for the confinement of low-energy electrons whereas an additional effect by the magnetic bottle potential reflecting electrons axially at the border of the ion trap cannot be excluded and is certainly important.

Our findings are considered to be of significant importance for future schemes to efficiently produce, trap, and $\operatorname{cool} \bar{H}$ in one region of space in a magnetic bottle configuration as planned e.g. in the next generation experiments within the ALPHA collaboration. In addition to the trapping of positrons by the ISCP of the antiprotons that seems be feasible according to our observations, axial drift tube potentials for the 
antiprotons might be dimensioned such that the $\bar{p}$ trapping electrostatic potential rises not earlier than the magnetic field, slightly different from our present EBIT configuration, such that the magnetic bottle effect is exploited more efficiently. If our present results are correct, $\mathrm{e}^{+}$and $\bar{p}$ would coexist in the same spatial region, the $\mathrm{e}^{+}$ being confined by the combined effect of the space charge of the $\bar{p}$ and of the magnetic bottle, the latter possibly allowing the number of stored $\mathrm{e}^{+}$to exceed the one of $\bar{p}$. Then, synchrotron cooling could be quite effective as is illustrated in Figure 5 (dashed lines with $\mathrm{N}_{\mathrm{e}}>\mathrm{N}_{\mathrm{i}}$ ) and relatively short cooling times of a few seconds are achieved along with a considerably enhanced recombination efficiency at low positron temperatures. In addition, as will be tested in our present trap, a strong laser could be used to stimulate recombination into high-lying states, which then in turn can be readily de-excited with further lasers, including a $\mathrm{Ly}_{\alpha}$ laser demonstrated recently [11]. Through the many viewports in our geometry, fluorescence radiation can be easily detected with reasonable solid angles.

Finally, we would like to point out that an EBIT configuration along with its viewports giving direct access to the centre of the trap shall be exploited in the future for precision spectroscopy of antiprotonic ions. Trapping low-energy $\bar{p}$ in the central drift tube and injecting any kind of atoms through an atomic jet (already installed in our present machines) should effectively generate antiprotonic ions ( $\bar{p}$-capture) which are efficiently trapped in the same region of space as the antiprotons. Emitted radiation after de-excitation of the bound $\bar{p}$ can be straight-forwardly detected through the viewports by large solid angle germanium detectors or by high-resolution x-ray crystal spectrometers. Envisioning cycle times of 10 seconds for loading of the trap with up to $10^{7} \bar{p}$ per shot as anticipated at the future Facility for Low-energy Antiproton and Ion Research (FLAIR) [12] at GSI might allow us to perform precision spectroscopy with effective signal rates of up to a few per second.

Moreover, we would like to emphasize, that new EBITs are presently under construction for charge breeding of radioactive ions to be generated at TRIUMF in Vancouver or at FAIR at GSI. Having trapped highly charged ions and $\bar{p}$ in a nested trap configuration, the ions in the central trapping region and the $\bar{p}$ at some neighbouring off-centre drift tube, would provide the unique possibility to let the $\bar{p}$ repeatedly collide with the highly charged radioactive heavy ions oscillating axially at well-controlled energies in the extended trapping region. $\bar{p}+\mathrm{A}^{\mathrm{q}^{+}} \rightarrow \mathrm{A}^{(\mathrm{q}-1)^{+* *}}$ resonant recombination to doubly excited combined electronic and antiprotonic states might provide a unique possibility to form and investigate well-defined antiprotonic states with only few bound electrons around as it has been recently demonstrated for pure doubly excited electronic states of He-, Li-, Be-, and B-like ions formed in our EBIT through dielectronic recombination [13].

Presently, we are designing a modified $\bar{p}$-EBIT and anticipate to start with tests by the end of the year, among them the above mentioned ones using ions and electrons to prove whether or not clouds of opposite charge can coexist in an EBIT configuration. 


\section{REFERENCES}

1. G. Gabrielse, N. S. Bowden, P. Oxley, A. Speck, C. H. Storry, J. N. Tan, M. Wessels, D. Grzonka, W. Oelert, G. Schepers, T. Sefzick, J. Walz, H. Pittner, T. W. Hänsch, E. A. Hessels, and (ATRAP Collaboration), Phys. Rev. Lett. 89, 213401 (2002)

2. M. Amoretti et al., Nature (London) 419, 456 (2002); M. C. Fujiwara et al. (ATHENA Collaboration), Phys. Rev. Lett. 92, 065005 (2004).

3. A. Mohri and Y. Yamazaki, Europhys. Lett. 63, 207 (2003).

4. C. H. Storry, A. Speck, D. L. Sage, N. Guise, G. Gabrielse, D. Grzonka, W. Oelert, G. Schepers, T. Sefzick, H. Pittner, M. Herrmann, J. Walz, T. W. Hänsch, D. Comeau, E. A. Hessels, and (ATRAP Collaboration), Phys. Rev. Lett. 93, 263401 (2004).

5. J. R. Crespo López-Urrutia, J. Braun, G. Brenner, H. Bruhns, C. Dimopoulou, I. N. Draganic, D. Fischer, A. J. González Martínez, A. Lapierre, V. Mironov, R. Moshammer, R. Soria Orts, H. Tawara, M. Trinczek, and J. Ullrich J. Phys: Conf. Series 2 42-51 (2004); J. R. Crespo López-Urrutia, B. Bapat, I. Draganic, B. Feuerstein, D. Fischer, H. Lörch, R. Moshammer, J. Ullrich, R. D. DuBois, and Y. Zou, Hyperfine Interactions 146-147, 109 (2003); J. R. Crespo López-Urrutia, et al., Phys. Scr. T 80 (1999).

6. A. Lapierre, U. Jentschura, J. R. Crespo López-Urrutia, J. Braun, G. Brenner, H. Bruhns, D. Fischer, A. J. González Martínez, V. Mironov, C. J. Osborne, G. Sikler, R. Soria Orts, H. Tawara,. Ch. Keitel, I. I. Tupitsyn, W. R. Johnson, J. Ullrich, A. Volotka, Phys. Rev. Lett. (submitted)

7. I. Draganic, J. R. Crespo López-Urrutia, R. DuBois, S. Fritzsche, V. M. Shabaev, R. S. Orts, I. I. Tupitsyn, Y. Zou, and J. Ullrich, Phys. Rev. Lett. 91, 183001 (2003).

8. J. Fajans, Physics of Plasmas, 101209 (2003)

9. L. Spitzer, Physics of Fully Ionized Gases, Interscience, New York 1956

10. U. Schramm, J. Berger, M. Grieser, D. Habs, E. Jäschke, G. Kilgus, D. Schwalm, A. Wolf, R. Neumann, and R. Schuch Phys. Rev. Lett. 67, 22-25 (1991); T. Schüssler, U. Schramm, T. Rüter, C. Broude, M. Grieser, D. Habs, D. Schwalm, and A. Wolf, Phys. Rev. Lett. 75, 802-805 (1995).

11. K.S.E. Eikema, J. Walz, and T.W. Hänsch, Phys. Rev. Lett. 83, 3828 (1999).

12. FLAIR Technical Design Report http://www-linux.gsi.de/ flair/.

13. A.J. González Martínez, A. Artemyev, J. Braun, G. Brenner, H. Bruhns, J.R. Crespo López-Urrutia, A. Lapierre, V. Mironov, J. Scofield, R. Soria Orts, H. Tawara, M. Trinczek, I. Tupytsin, and J. Ullrich, Phys. Rev. Lett. (accepted) 